\title{
Optical Imaging of Odor-Evoked Glomerular Activity Patterns in the Antennal Lobes of the Ant Camponotus rufipes
}

\author{
C.G. Galizia ( $\varangle)$, R. Menzel \\ Institut für Neurobiologie, Freie Universität Berlin, Königin Luise Strasse 28-30, D-14195 Berlin, Germany \\ e-mail: galizia@zedat.fu-berlin.de, Tel.: +49-30-8386454, Fax: +49-30-8385455 \\ B. Hölldobler \\ Universität Würzburg, Institut für Zoologie, Theodor-Boveri-Zentrum für Biowissenschaften (Biozentrum), \\ Am Hubland, D-97074 Würzburg, Germany
}

Received: 10 March 1999 / Accepted in revised form: 5 July 1999

\begin{abstract}
Ants have a well developed olfactory sense, which they need both for the perception of environmental chemicals, and for a highly sophisticated intraspecific communication system based on pheromones. The question arises therefore as to how different odors are coded in the antennal lobe, the first central neuropil to process olfactory information. We measured odor-evoked activity patterns using in vivo neuropil calcium recording in the antennal lobe of the ant Camponotus rufipes. We found that (a) odors elicit focal activity spots (diameter ca. $20 \mu \mathrm{m}$ ) which most probably represent the olfactory glomeruli; (b) different odors are coded in odor specific patterns of such activated spots, and a particular spot can participate in the pattern for different odors; (c) calcium increased in the activated spots within the 2-s stimulation period and slowly declined thereafter.
\end{abstract}

\section{Introduction}

Olfaction is the primary sense for many animal species. In social insects, such as bees and ants, odors are important for a variety of behavioral activities: location of food sources (e.g., by smelling specific plant or prey odors), intraspecific communication (e.g., when following a trail marked with a specific pheromone), interspecific communication (e.g., in symbiotic interactions with other species), and for development (e.g., when a queen of bees or ants signals her presence and thereby affects the reproduction of the worker caste; Hölldobler and Wilson 1990). A number of these chemicals must to be identified unambiguously by the olfactory system. Some are genetically predictable because as pheromone substances their production is coded in the same genome as the receptor cells which they stimulate, others are environmentally predictable, such as specific food odors, and again others are unpredictable, such as flower odors which change with season and geographic range.

The antennal lobe (AL) is the primary olfactory neuropil in the insect brain. It receives afferent input from the receptor cells on the antenna and relays this information via the projection neurons to higher order brain centers, such as the mushroom bodies and the lateral protocerebrum. In the AL synaptic contacts are limited to neuropil structures of diameter between 20 and $50 \mu \mathrm{m}$, called glomeruli, which surround a central coarse neuropil. Local interneurons interconnect the glomeruli. These basic properties of insect ALs have been the subject of several reviews (Boeckh and Tolbert 1993; Hildebrand and Shepherd 1997; Masson and Mustaparta 1990; Rospars 1988; Stocker 1994).

The number of glomeruli varies greatly between species, ranging from 43 in Drosophila melanogaster (Laissue et al. 1999) to 1000 in Schistocerca gregaria (Rospars 1988). Even within hymenopteran species the number of glomeruli varies greatly: Apis mellifera has 160 and Vespa crabro 1000 glomeruli. The question arises therefore as to whether the functional organization of glomeruli is similar in different species.

It has been shown by using in vivo optical recording of intracellular calcium concentration that odors evoke characteristic and stimulus-specific glomerular activity patterns in the ALs of honeybees (Galizia et al. 1997, 1998; Joerges et al. 1997). In this study we investigated the possibility of applying the 
same method to ALs of the ant Camponotus rufipes. This formicine species is quite common in South America. Part of its chemical communication system is well studied (Übler et al. 1995). Therefore an analysis of its olfactory system appears timely.

\section{Materials and Methods}

\section{Animals}

All animals came from the stock reared at the University of Würzburg and are the progeny of a mated female from El Bagnol, Argentina, collected in October 1996. They were fed corpses of various insect species and honey diluted in water. Mediumsized individuals where used in all experiments. This contribution is based on the measurement of 49 individuals, 7 of which showed stimulus-correlated activity. Only the latter were included in the data analysis.

\section{Calcium Imaging}

For functional optical imaging the method described for honeybees (Galizia et al. 1997, 1998) had to be adapted to the ant. The ants were cooled in order to anesthetize them and fixed with dental wax in a custom made recording chamber of Plexiglas. A window was cut open between the eyes and behind the antennae. Then tracheae and muscles were removed from the head capsule, taking great care not to damage the optical or antennal nerves. Using a sharpened and bent needle (minute insect pin with shaft diameter of $150 \mu \mathrm{m}$ ) the neurolemma above the ALs was gently ripped, without changing the appearance of the AL itself (i.e., if at this point the AL looked as if the glomeruli were popping out of it by excess internal pressure, the preparation was discarded). This neurolemma peeling proved to be the most delicate step in the preparation. Then $50 \mu \mathrm{l}$ dye solution (50 mg calcium green $2 \mathrm{AM}$, Molecular Probes; dissolved first in $50 \mu \mathrm{l} 20 \%$ pluronic plus DMSO, and this stock solution diluted with $800 \mu \mathrm{l}$ physiological saline solution, see below) was applied to the head capsule. The preparation was covered with a cover-slip, and placed in a cool $\left(10^{\circ} \mathrm{C}\right)$, dark container for $1 \mathrm{~h}$. After this incubation time the brain was rinsed in fresh physiological saline solution $(130 \mathrm{mM} \mathrm{NaCl}, 6 \mathrm{mM} \mathrm{KCl}, 4 \mathrm{mM} \mathrm{MgCl} 2,5 \mathrm{mM}$ $\mathrm{CaCl}_{2}, 160 \mathrm{mM}$ sucrose, $25 \mathrm{mM}$ glucose, $10 \mathrm{mM}$ HEPES, pH 6.7, $500 \mathrm{mOsmol}$ ). Then a clean needle (shaft diameter $150 \mu \mathrm{m}$ ) was inserted between subesophageal ganglion and protocerebrum to stabilize mechanically the brain, and the preparation was placed under the microscope.
Images were taken through $\mathrm{a} \times 40$ air $\mathrm{NA}=0.6 \mathrm{ob}$ jective. Each measurement consisted of 40 measured frames, at a rate of two frames per second. Exposure time for each frame was $240 \mathrm{~ms}$. Odorant stimulus was given from the 9 th to the 13th frames. Spatial resolution was $51 \times 51$ pixels (binned on chip from the $512 \times 512$ pixels of the cooled CCD camera, Photometrics $\mathrm{CH} 250 \mathrm{~A}$ ), spanning $250 \times 250 \mu \mathrm{m}$ of the AL. Each binned pixel corresponds to $5 \times 5 \mu \mathrm{m}$. From the raw fluorescence intensity data signals were calculated as $\Delta \mathrm{F} / \mathrm{F}$. The mean fluorescence over the entire 40 frames was taken, for each pixel, as an estimate for F. Dark current of the CCD camera was measured before each experiment (i.e., before each measured ant) and subtracted from the measured fluorescence intensities. Odors were delivered with a computer controlled olfactometer (Galizia et al. 1997). For each odor $4 \mu \mathrm{l}$ of the pure substance was placed on a piece of filter-paper $\left(1 \mathrm{~cm}^{2}\right)$ and the filter paper placed in a $1 \mathrm{ml}$ plastic syringe which in turn was inserted into the olfactometer. Odors used were: citral, 4-methyl-3-heptanol, 1-hexanol, hexanal, 2-methyl-3-buten-2-ol, 4-methylpyrrole-2-carboxylic acid-methylester (dissolved in hexane), menthol (dissolved in hexane), $n$-undecane. All substances were from Sigma, Deisenhofen, Germany.

\section{Results}

\section{Spatial Pattern of Activated Glomeruli}

Stimulation with odors leads to specific spatial activity patterns in the AL that can be measured with calcium imaging. Figure 1 presents the response patterns to eight different odors. The activity patterns consist of several discrete foci, which differ from odor to odor, although the same focus may be activated by more than one odor. The activated foci are generally spatially separated from each other, which suggests that contiguous glomeruli do not necessarily have similar response profiles. The response to hexanal appears to be more widespread, with a central active band on the AL. The diameter of the foci is about $20 \mu \mathrm{m}$, which is in the range of the diameter of olfactory glomeruli. For every given odor most of the AL remained inactive. The elicited patterns matched for repeated stimulation with the same odor (data not shown), as has also been reported for glomeruli in honeybees (Joerges et al. 1997; Galizia et al. 1998). All these observations together argue for the activity spots to correspond to olfactory glomeruli.

No qualitative difference was found between the patterns elicited by $n$-undecane (an alarm phero- 

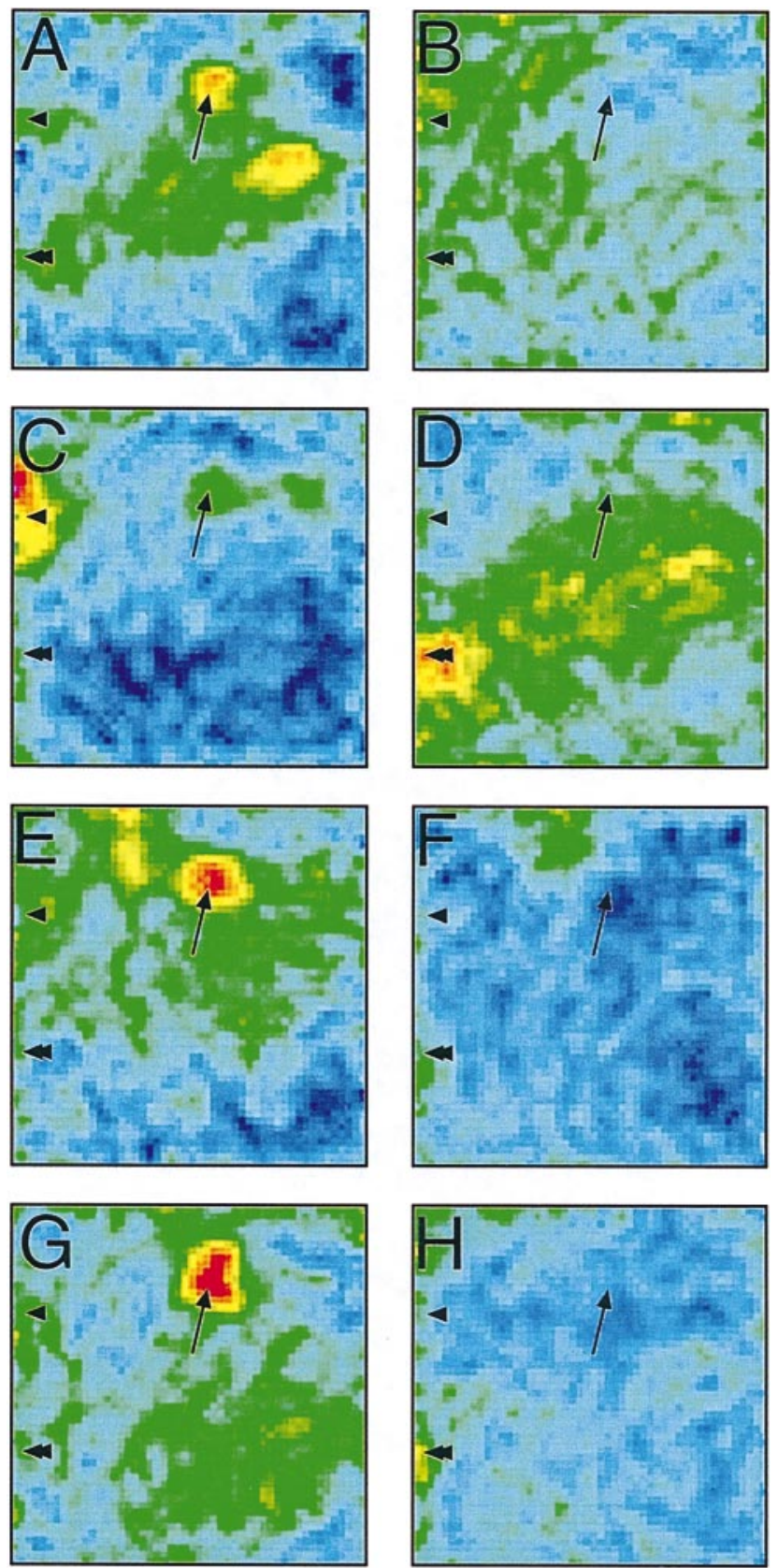

$0 \%$ $\Delta F / F$

0.7

mone in C. ligniperdus and several other Camponotus species, and probably also in C. rufipes; Hölldobler and Wilson 1990), and the other substances.

\section{Temporal Pattern of Odor Evoked Activity}

Upon stimulus delivery there was a significant increase in intracellular calcium in the activated glom-
Fig. 1A-H. False-color coded activity patterns in the AL of C. rufipes as response to different odor stimuli. False-color bar (at bottom) color-code for values of $\Delta \mathrm{F} / \mathrm{F}$ ranging from $0 \%$ to $0.73 \%$. The antennal nerve is pointing up, image size is $250 \times 250 \mu \mathrm{m}$. Each panel shows the response to a different odor. Most odors are common plant odors, such as citral, 1-hexanol, and hexanal, and may also act as pheromones in some species. A) Citral, an alarm pheromone in some ant species. B) 4-Methyl-3-heptanol, a substance used as trail pheromone in some ant species, and found in the mandibular gland in others. C) 1-Hexanol, an alarm pheromone in some species. D) Hexanal, also an alarm pheromone in some species. E) 2-Methyl-3-buten-2-ol, an alarm pheromone in Vespa crabro. F) 4-Methylpyrrol-2-carboxylacid-methylester, a trail pheromone for various species of leaf-cutting ants. G) Menthol. H) $n$-Undecane, an alarm pheromone in many Camponotus species. Some glomeruli are marked with arrow, arrowhead, or double arrowhead to ease comparison. Note the overlap in the elicited patterns. For example, citral, 2-methyl-3-buten-2-ol and menthol all elicit activity in the position marked (arrow)

eruli, which reached its maximum within $2-3 \mathrm{~s}$. Figure 2 shows the temporal activation pattern for several glomeruli for different odor stimuli. The signal decayed to baseline within 5-8 s. Maximum response amplitude was about $0.7 \% \Delta \mathrm{F} / \mathrm{F}$. Note that in different glomeruli the response to the various odors has a different time latency (Fig. 2). Also note the different response patterns in Fig. $2 \mathrm{~B}$ and Fig. 2D, which represent two positions at a distance of $20 \mu \mathrm{m}$, i.e., two neighboring glomeruli.

\section{Discussion}

\section{Methodological aspects of calcium imaging in the ant $A L$}

To analyze the network properties of brains it is necessary to measure the activity in entire neuropils. Here we report the successful calcium imaging of an entire neuropil in a living insect brain other than the honeybee. The transferability of a method from one species to another is not a trivial matter, and often entails surprises. For example, radioactively labeled 2-deoxyglucose staining has been used to measure activity in Drosophila melanogaster (Rodrigues 1988) and has recently been used in the blowfly Calliphora vicina (Distler 1998), but the same method does not work in the cockroach Periplaneta americana (P. Distler, personal communication), possibly because of differences in glucose metabolism.

In adapting the technique to the ant we had to introduce a number of changes. The two most important differences are the following: in the ant we had to damage the neurolemma mechanically using a small pin. Failure to do so invariably resulted in insufficient or absent loading of the dye. The neurolemma in the ant is mechanically much more resistant than 

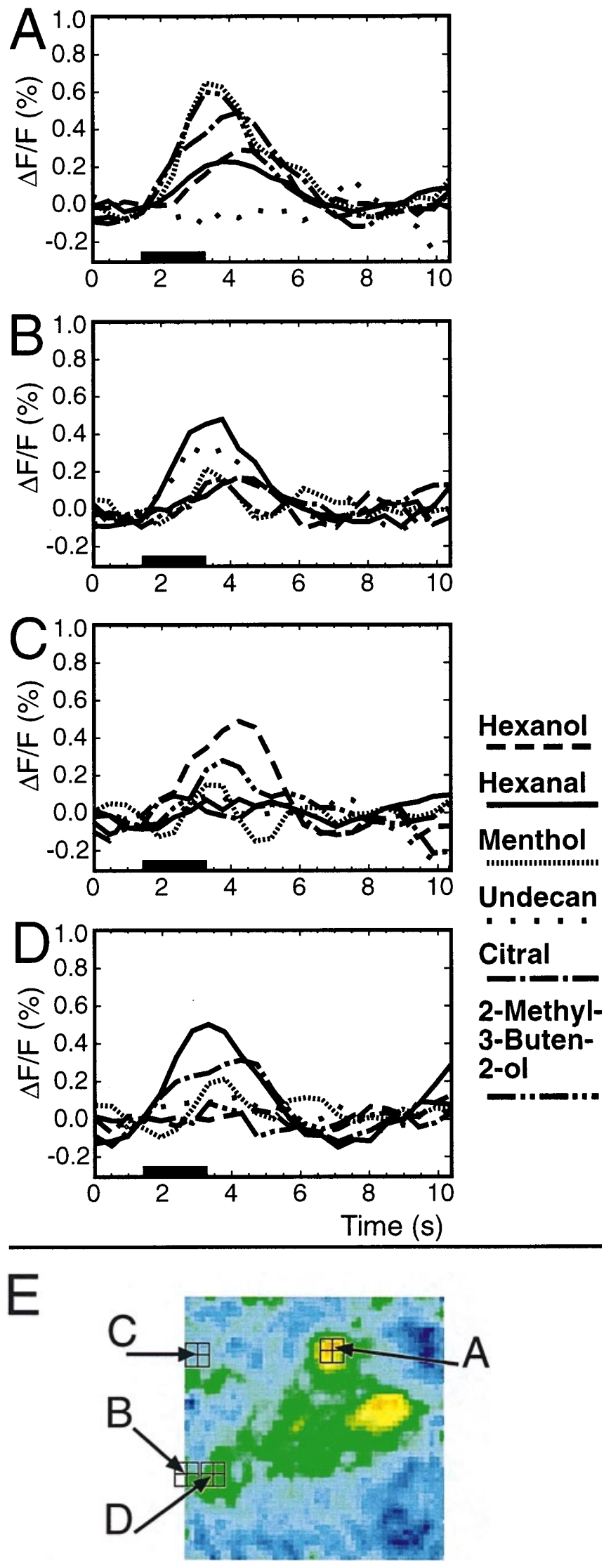

Fig. 2A-E. Time course of the signals in individual glomeruli. A-D) For each glomerulus the responses to six different odors are plotted (see figure). Relative fluorescent changes $(\Delta \mathrm{F} / \mathrm{F})$ corrected against bleaching by subtracting the air control time-course are plotted against time (s). Black bar stimulus. The position of the glomeruli shown is given in $\mathrm{E}$. Note that $\mathrm{B}$ and $\mathrm{D}$ are direct neighbors. For each glomerulus a square of $5 \times 5$ pixels $(25 \times 25 \mu \mathrm{m})$ was averaged; for glomerulus $\mathrm{B}$ the square was $3 \times 5$ pixels

that in the honeybee, making it likely that it also forms a more efficient blood-brain barrier. Furthermore, we had to stabilize the brain with a pin. In $C$. rufipes the brain almost floats in the head capsule and is attached to the very thin optical and olfactory nerves. Since a stable preparation and the lack of movement are compulsory for imaging purposes, the brain had to be fixed artificially.

The signals in $C$. rufipes were much smaller than those in $A$. mellifera (maximum signals of $0.8 \% \Delta \mathrm{F} /$ $\mathrm{F}$ in the ant against generally $2-3 \% \Delta \mathrm{F} / \mathrm{F}$, and sometimes up to $5 \%$, in the bee). This is due partly to a higher background caused by tissue autofluorescence, which leads to the meaningful light intensity changes riding on top of a strong background light. This reduces the relative light changes, and therefore the signals measured as $\Delta \mathrm{F} / \mathrm{F}$.

Hexanol Hexanal

Menthol

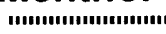
Undecan

Citral

2-Methyl3-Buten2-ol

\section{Spatiotemporal activity patterns}

The activity patterns observed in the olfactory glomeruli are shaped by the response properties of the receptor neurons innervating them, and by interglomerular connections. It is unknown whether in ants (or in any other insect) each glomerulus obtains input from only one or from several receptor neuron types, and whether each receptor neuron type exclusively innervates one glomerulus or more than one. However, if more than one glomerulus receives identical input, one would expect a high correlation in the activity responses of groups of glomeruli. This 
was not seen in our measurements: although each odor elicited activity in several glomeruli, no group of glomeruli responded in a correlated manner to the tested odors. The data are not yet conclusive. Since in $C$-rufipes the glomeruli lie beneath each other, it may be that glomeruli with similar response profiles were out of focus, or masked by more superficial glomeruli, and thus not visible in our activity measurements. Furthermore, the lateral, medial, and posterior side of the AL could also not be imaged.

The other two possibilities (innervation of each glomerulus with one receptor type, or with a unique combination of different receptor types) would lead to activity patterns which are comparable to those which we found, assuming that the response profile of a receptor neuron is a complex pattern: each odor elicits a unique pattern of several activated glomeruli. For example, in Fig. 1, the glomerulus marked with an arrow responds to citral, 2-methyl-3-buten2-ol and menthol, as well as (weakly) to 1-hexanol. This means that either one receptor type has a broad response spectrum encompassing all of these odors, or that several receptor types innervate this glomerulus, thus leading to this complex response. A decision between these two hypotheses must rely on physiological measurements from single receptors, receptor neuron mapping, and/or on functional staining of their axons.

The temporal properties found in ants are comparable with our findings in honeybees: in both species there is a strong increase within the first two seconds, followed by a slower decline. Also, the response to a given odor is of different intensity in different glomeruli, and its onset may differ by one or two frames $(0.5-1 \mathrm{~s})$. Delayed responses are usually of weak intensity (see Fig. 2).

Summing up, the measurements from $C$. rufipes are in good agreement with what we find in honeybees. We conclude that the basic physiological organization is very similar in these two species. Studying the olfactory code in ants is rewarding for two reasons: first, ants have a plethora of different pheromones. Some of these differ in the behavior which they elicit depending on their absolute concentration (Hölldobler and Wilson 1990). However, as in worker honeybees, the ALs do not have any morphologically evident structures devoted to pheromone processing. The data presented here suggest that pheromonal and nonpheromonal processing are also functionally indistinguishable at the level of the AL. Behaviorally, pheromones lead to stereotyped responses, while environmental odors lead to responses shaped by experience. This difference may be a task of structures upstream of the AL, as the mushroom bodies or the lateral protocerebrum. A comparison between closely related ant species, which have clearly separated pheromonal signals, but very similar morphology, would be instructive in this context: does a glomerular pattern code for a behavioral meaning (which would mean that the afferent innervation is different between the observed species), or does it merely reflect the chemical composition of the odors, irrespective of their meaning, which would imply that the differences start upstream, possibly at the level of the projection neurons.

Acknowledgements We thank Malu Obermayer for advisory and histological help, Astrid Klawitter for help in maintaining the ants in captivity, and Kerry Delaney for helpful discussions and insights into optical imaging. This research was supported by Deutsche Forschungsgemeinschaft ME 365/21.

Boeckh J, Tolbert LP (1993) Synaptic organization and development of the antennal lobe in insects. Microsc Res Tech 24:260-280

Distler PG, Bausenwein B, Boeckh J (1998) Localization of odorinduced neuronal activity in the antennal lobes of the blowfly Calliphora vicina: a $\left[{ }^{3} \mathrm{H}\right] 2$-deoxyglucose labeling study. Brain Res $805: 263-266$

Galizia CG, Joerges J, Kuettner A, Faber T, Menzel R (1997) A semiin-vivo preparation for optical recording of the insect brain. J Neurosci Methods 76:61-69

Galizia CG, Nägler K, Hölldobler B, Menzel R (1998) Odour coding is bilaterally symmetrical in the antennal lobes of honeybees (Apis mellifera). Eur J Neurosci 10:2964-2974

Galizia CG, Sachse S, Rappert A, Menzel R (1999) The glomerular code for odor representation is species specific in the honeybee Apis mellifera. Nat Neurosci 2:473-478

Hildebrand JG, Shepherd GM (1997) Mechanisms of olfactory discrimination: converging evidence for common principles across phyla. Annu Rev Neurosci 20:595-631

Hölldobler B, Wilson EO (1990) The ants. Springer, Berlin Heidelberg New York

Joerges J, Kuettner A, Galizia CG, Menzel R (1997) Representations of odours and odour mixtures visualized in the honeybee brain. Nature 387:285-288

Johnson BA, Woo CC, Leon M (1998) Spatial coding of odorant features in the glomerular layer of the rat olfactory bulb. J Comp Neurol 393:457-471

Laissue PP, Reiter C, Hiesinger PR, Halter S, Fischbach K-F, Stocker RF (1999) Three-dimensional reconstruction of the antennal lobe in Drosophila melanogaster. J Comp Neurol 405:543-552

Masson C, Mustaparta H (1990) Chemical information processing in the olfactory system of insects. II. Central nervous system, development, and plasticity. Physiol Rev 70:215-245

Mori K, Mataga N, Imamura K (1992) Differential specificities of single mitral cells in rabbit olfactory bulb for a homologous series of fatty acid odor molecules. J Neurophysiol 67:786-789

Rodrigues V (1988) Spatial coding of olfactory information in the antennal lobe of Drosophila melanogaster. Brain Res 453:299-307

Rospars JP (1988) Structure and development of the insect antennodeutocerebral system. Int J Insect Morphol Embryol 17:243-294

Stocker RF (1994) The organization of the chemosensory system in Drosophila melanogaster: a review. Cell Tissue Res 275:3-26

Übler E, Kern F, Bestmann HJ, Hölldobler B (1995) Trail pheromone of two formicine ants, Camponotus silvicola and C. rufipes (Hymenoptera: Formicidae). Naturwissenschaften 82:523-529 